Trauma Berufskrankh 2012 · 14[Suppl 3]:335-343 DOI 10.1007/s10039-011-1800-6

Online publiziert: 24. März 2012

(c) Springer-Verlag 2012

A. Prokop ${ }^{1} \cdot$ C. Koukal $^{1} \cdot$ R. Dolezych ${ }^{2} \cdot$ M. Chmielnicki ${ }^{1}$

${ }^{1}$ Unfallchirurgie, Kliniken Sindelfingen, Klinikverbund-Südwest, Sindelfingen

${ }^{2}$ Medizincontrolling, Kliniken Sindelfingen, Klinikverbund-Südwest, Sindelfingen

\title{
Minimalinvasive Versorgung von thorakolumbalen Wirbelfrakturen
}

\section{Möglichkeiten und Grenzen}

Die wesentliche Entwicklung in der Traumatologie der letzten Jahre waren minimalinvasive Operationstechniken. Patienten können durch Vermeidung großer Zugänge ohne Schädigung der angrenzenden Weichteile und der Muskulatur schneller mobilisiert werden und leiden weniger unter Schmerzen. Somit sind aufwendige Operationen auch bei betagten Patienten möglich.

Gerade in Anbetracht der demografischen Entwicklung werden wir es zunehmend mit alten Patienten und begleitender Osteoporose zu tun haben. Bereits heute sind $1 / 3$ aller stationären Patienten eines unfallchirurgischen Versorgungskrankenhauses über 70 Jahre alt [14]. Somit sind neben möglichst kleinen Schnitten bei der Operation auch besondere Anforderungen an moderne Implantate $\mathrm{zu}$ stellen, die die notwendige Stabilität auch im osteoporotischen Knochen erlauben.

Auch im Bereich der Wirbelsäulenchirurgie war eine rasante Entwicklung in den Techniken und den Implantaten zu verzeichnen, sodass die minimalinvasive Versorgung von Wirbelbrüchen in den letzten 5 Jahren von einer Außenseiterrolle zum Standard mutierte.

Neben den vielen Vorteilen sind minimalinvasive Eingriffe aber technisch anspruchsvoller und bedürfen eines ausgefeilten Instrumentariums und einer Lernkurve des Operateurs. Die intraoperative Visualisierung ist oft nur radiologisch möglich, und die Implantate sind in der Regel deutlich teurer.
Gerade in der Frakturversorgung bestehen sowohl im In- und Ausland als auch bei Unfallchirurgen und Orthopäden oder Neurochirurgen z. T. unterschiedliche Behandlungskonzepte. In unserem Haus wurden die minimalinvasiven Techniken (perkutane Fixateure, endoskopische ventrale Spondylodesen und perkutane Kyphoplastien) von Beginn an betrieben und über Jahre hinweg die Indikationen anhand der eigenen Erfahrungen verifiziert. Wir berichten daher nachfolgend über unsere persönlichen Erfahrungen und altersadaptierten Behandlungsstrategien $[7,8,9,10,11,12,13,14,15$, 16]. Unabhängig von anderen Meinungen, möchten wir die Frage beantworten: Wann führen wir in welchem Alter was und warum durch?

\section{Dorsale Instrumentierung bei instabilen Frakturen}

Wenn die Hinterkante der Wirbel mitbetroffen oder gegen den Spinalkanal imprimiert ist, bei instabiler Fraktur und bei vorhandenem Risiko eines neurologischen Ausfalls oder einer Kyphose [Wolter-Klassifikation > Typ AB, AO-Klassifikation: A3-, B- und C-Frakturen (AO: Arbeitsgemeinschaft für Osteosynthesefragen)], ist die dorsale Instrumentierung der Goldstandard zur Erstversorgung.

\section{Offene Instrumentierung}

Bei der offenen dorsalen Instrumentierung von instabilen Wirbelsäulenfrakturen kommt es bei der Ablösung der paravertebralen Muskulatur immer zu Blutungen und im späteren Verlauf zur Narbenbildung und zu Funktionsstörungen. Große Schnitte über den Dornfortsätzen bereiten Schmerzen und führen später zu kosmetischen Beeinträchtigungen.

Je länger die Retraktion der Muskulatur unter der Operation dauert, desto schlechter ist die postoperative Muskelfunktion. So konnte Gejo et al. [4] prospektiv 20 Patienten nach dorsalen Instrumentierungen dynamisch und in der Magnetresonanztomographie (MRT) untersuchen. Bei einer Operationszeit über 80 min wurden in der Muskulatur im MRT postoperativ signifikant mehr Einblutungen und Vernarbungen beobachtet. Bei kinematischen Untersuchungen der Muskulatur mittels Dynamometer ergab sich eine deutliche Beeinträchtigung deren Funktion.

Sihvonen et al. [17] untersuchten Patienten, die nach dorsalen Eingriffen über postoperative Rückenschmerzen klagten, radiologisch, neurophysiologisch und anhand von Muskelbiopsien. Sie konnten zeigen, dass es durch den Zugang zu In-

Alle in der Arbeit beschriebenen Operationen sind als Videos unter youtube.com: Stichwort „Kliniken Sindelfingen“ abrufbar. 

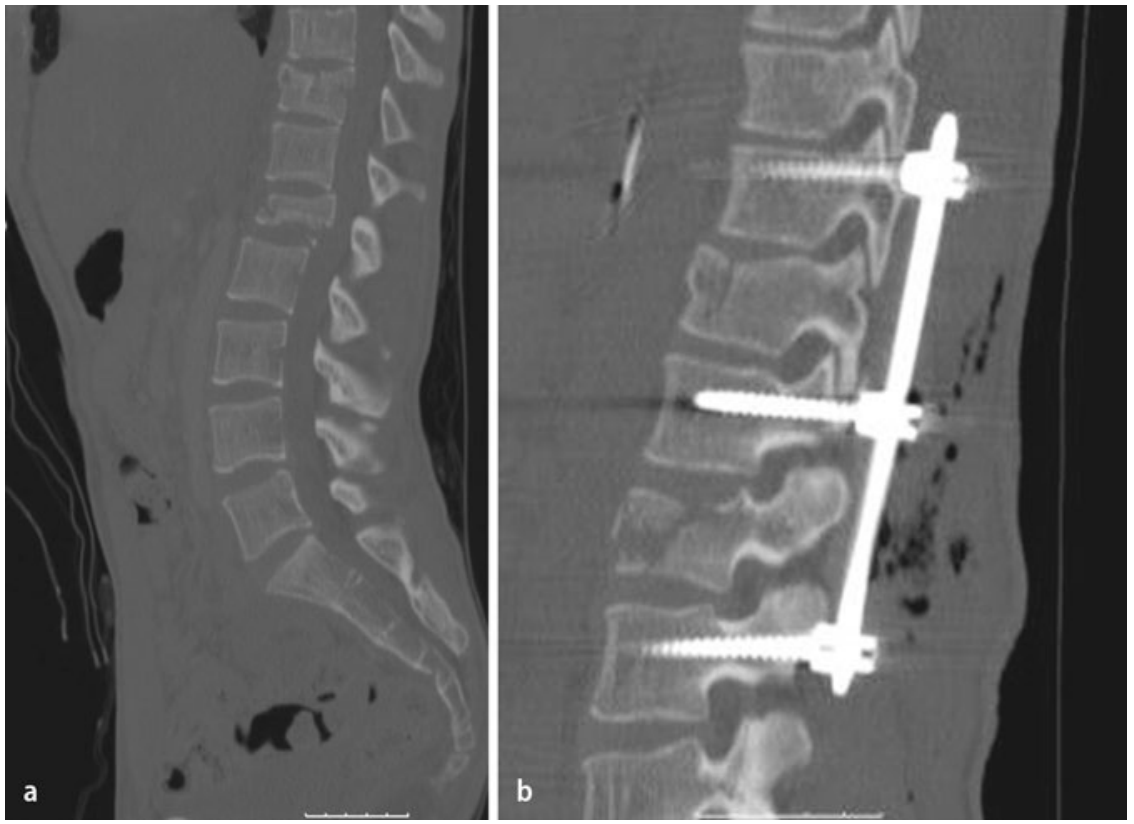

Abb. $1 \Delta$ Patient mit instabiler LWK1- (LWK: Lendenwirbelkörper) und BWK11-Fraktur (BWK: Brustwirbelkörper), a präoperative seitliche Computertomographie (CT), b postoperative seitliche $\mathrm{CT}$ nach Versorgung mit Longitude-Fixateur

nervationsstörungen der Muskulatur mit Substanzverlusten kam, die zu messbaren schmerzhaften Funktionseinschränkungen führten.

\section{Minimalinvasive Versorgung}

\section{Technik}

Wir setzen als perkutane Verfahren Fixateure der Firma Medtronic ein, wobei 2 unterschiedliche Systeme zur Verfügung stehen. Beim Sextant-Fixateur werden polyaxiale Schrauben benutzt und der Längsträgerstab über einen aufgesetzten Zielbügel in die Schrauben sehr leicht zielsicher perkutan eingeschoben. Die Instrumentierung ist einfach und schnell, aber eine Reposition ist nur eingeschränkt im ventralen Durchhang möglich. Beim Longitude-Fixateur werden mono- oder polyaxiale Schrauben über perkutane Zieldrähte positioniert und der Längsträger manuell perkutan eingefädelt. Die Instrumentierung ist etwas schwieriger, dafür aber über mehrere Etagen anwendbar. Bei den monoaxialen Schrauben kann neuerdings über einen aufsetzbaren $\mathrm{Re}$ traktor zusätzlich distrahiert werden.

In einer ersten Pilotstudie versorgten wir im Jahr 200836 instabile Wirbelsäulenfrakturen mit der minimalinvasiven Technik und verglichen diese mit $40 \mathrm{~Pa}$ - tienten, die in der offenen Technik mit einem Fixateur der Firma Synthes (USS II) versorgt worden waren. Das Durchschnittsalter lag bei 61 Jahren (22-88 Jahren). Nach der AO-Klassifikation handelte es sich vornehmlich um A3.3-Frakturen. Die mittlere Schnitt-Naht-Zeit betrug mit der minimalinvasiven Technik 42 min (14-96 min) bei einer Durchleuchtungszeit von 3:35 min (1:50-7:00 min). Der Blutverlust lag bei im Mittel $20 \mathrm{ml}$. Alle Patienten standen am ersten postoperativen Tag auf und wurden mobilisiert. Die Schmerzen, bemessen nach der visuellen Analogskala, konnten im Mittel von 6,1 auf 1,4 Punkte gesenkt werden. In $2 \%$ der Fälle wurden asymptomatische Schraubenfehllagen gesehen, die nicht revidiert werden mussten. Die Krankenhausliegezeit betrug seinerzeit 11 Tage. Die durchschnittliche Operationszeit bei einem Kontrollkollektiv mit 40 offen operierten Patienten lag dagegen bei $91 \mathrm{~min}$ und war somit um 39 min länger. Der mittlere Blutverlust betrug $720 \mathrm{ml}(+700 \mathrm{ml})$ und die mittlere Liegezeit 15 Tage (+4 Tage). Nicht korrekturbedürftige Schraubenfehllagen wurden in $4 \%$ aller Schrauben beobachtet $[7,8]$. Seit dieser Zeit kamen in unserem Haus ausschließlich die perkutanen Techniken zum Einsatz. Die Pedikulierung kann radiologisch a.-p. (Schieß- scheibentechnik) und seitlich gut kontrolliert werden, was die Rate der Schraubenfehllagen verringert.

Bei jungen Patienten unter 60 Jahren mit guter Knochenstruktur kommen monoaxiale Schrauben und der Longitude-Fixateur zum Einsatz, um eine optimale Reposition zu ermöglich. Bei Mehretagenfrakturen nutzen wir den Longitude-Fixateur mit polyaxialen Schrauben, um einen Halt der Längsträger über alle Schrauben zu erreichen (• Abb. 1). Nachdem die Längsträger als Toplader eingeschoben sind, werden sie über die Schraubenaufsätze nach unten in die Pedikelschrauben gepresst. Bei vorliegender Osteoporose ist in diesem Moment oft ein axiales Herausziehen der Pedikelschrauben aus dem Wirbelkörper zu beobachten, sodass wir in diesen Fällen lieber auf den Sextanten mit einem einschwenkbaren Längsträger ohne axialen Zug auf die Schrauben zurückgreifen. Bei Patienten über 60 Jahren und instabilen Frakturen wird demzufolge der Sextant verwendet. Polyaxiale Schrauben mit distaler Fensterung erlauben seit neuerer Zeit eine zusätzliche Zementaugmentierung der Schrauben im osteoporotischen Knochen. Eine zusätzliche Distraktion ist hier allerdings nicht oder nur sehr eingeschränkt möglich. Die Reposition erfolgt fast ausschließlich im ventralen Durchhang.

Bei ergänzender Neurologie wird über dem betroffenen Segment eröffnet und eine Hemilaminektomie ein- oder beidseits oder eine Laminektomie durchgeführt. Für diese Fälle stehen Querverbinder als ergänzende Stabilisatoren zur Verfügung. Die Pedikelschrauben werden aber ebenfalls perkutan eingebracht [2].

\section{Ergebnisse}

Vom 01.01.2008 bis zum 01.08.2011 wurden in unserem Haus nach obigen Vorgaben 309 Patienten mit instabilen Wirbelfrakturen perkutan dorsal instrumentiert, wobei 9 verschiedene Operateure die Eingriffe durchführten. Die Patienten, 156 Männer und 153 Frauen, hatten ein Durchschnittsalter von 63 Jahren. Es wurden Frakturen von BWK3 (Brustwirbelkörper 3) bis LWK5 (Lendenwirbelkörper 5) stabilisiert. In 2/3 der Fälle lagen sie zwischen BWK11 und LWK2. In 5\% der Fälle wurden zusätzliche Hemi- oder La- 
minektomien ergänzt. Die Schnitt-NahtZeit inklusive Mehretagenversorgungen und Laminektomien betrug im Mittel 66 min (14-90 min). Die Patienten blieben im Durchschnitt 13 Tage (3-22 Tage) stationär, mit über die Jahre deutlich fallender Tendenz und umso kürzerer Aufenthaltsdauer je jünger die Patienten waren. Neurologische Ausfälle als Folge der Operation traten nicht auf.

Im osteoporotischen Knochen bei Patienten über 60 Jahre wurde wiederholt ein Durchschneiden des Fixateurs beobachtet. In einer Serie in den Jahren 2007 und 2008 wurden 61 Patienten über 60 Jahre mit einem Altersdurchschnitt von $73 \pm 8$ Jahren (60-88 Jahren) mit instabilen Wirbelfrakturen am thorakolumbalen Übergang mit einem perkutanen Fixateur interne versorgt. Präoperativ, postoperativ und nach 6 Wochen funktioneller korsettfreier Behandlung wurde der Kyphosewinkel im seitlichen Röntgenbild bestimmt und im Student-t-Test verglichen. In 7 Fällen wurden zementaugmentierte Schrauben verwendet. Der Kyphosewinkel betrug präoperativ $13,9 \pm 9,4^{\circ}$ und postoperativ $8,1 \pm 6,7^{\circ}$. Somit konnte durch die Operation eine Aufrichtung von $5,8 \pm 5,9^{\circ}(\mathrm{p}<0,001) \mathrm{er}-$ reicht werden. Innerhalb der ersten 6 Wochen postoperativ kam es jedoch wieder zur Sinterung um $7,7 \pm 7,4^{\circ}(p<0,001)$, bedingt durch das Durchschneiden der Schrauben durch den Wirbelkörper. Die Schrauben-Stab-Schrauben-Verbindung blieb in allen Fällen ohne signifikante Änderungen konstant. Bei den Versorgungen mit zementaugmentierten Schrauben kam es nicht zu Repositionsverlusten, sodass wir seit dieser Zeit eine Zementaugmentation der Pedikelschrauben vornehmen [2]. Kommt es dennoch zu Anschlussfrakturen, muss der Fixateur verlängert werden. In einem Fall kam es bei einem 81-Jährigen mit instabiler BWK12Fraktur trotz zementiertem Fixateur zum Ausbruch mit Anschlussfrakturen und $60^{\circ}$ Kyphose. Es wurde minimalinvasiv revidiert, der Fixateur wurde verlängert, und es wurden Kyphoplastien der Anschlussfrakturen vorgenommen (• Abb. 2) - mit guter Befindlichkeit des Patienten $12 \mathrm{Mo}$ nate nach dem Revisionseingriff.

Trauma Berufskrankh 2012 · 14[Suppl 3]:335-343 DOI 10.1007/s10039-011-1800-6

(c) Springer-Verlag 2012

\section{A. Prokop · C. Koukal · R. Dolezych · M. Chmielnicki \\ Minimalinvasive Versorgung von thorakolumbalen Wirbelfrakturen. Möglichkeiten und Grenzen}

\section{Zusammenfassung}

Minimalinvasive Operationen bei Wirbelfrakturen bedeuten weniger Zugangsmorbidität, geringere postoperative Schmerzen und raschere Mobilisierung der Patienten, sind aber technisch anspruchsvoller, bedürfen eines ausgefeilten Instrumentariums und einer Lernkurve des Operateurs. Die intraoperative Visualisierung ist oft nur radiologisch möglich, die Implantate sind meist deutlich teurer. In Abhängigkeit vom Alter des Patienten und der Fraktur entwickelten wir bei knapp 1000 Frakturversorgungen in den letzten 3,5 Jahren ein differenziertes Behandlungskonzept: Instabile Frakturen mit Beteiligung der Hinterkante werden von dorsal mit einem Fixateur perkutan stabilisiert. Bei unter 60-Jährigen kommen monoaxia- le Schrauben, bei über 60-Jährigen polyaxiale Sextantensysteme, jeweils mit eingeschobenen Längsträgern, zum Einsatz. Verbleibt nach dorsaler Versorgung ein Defekt des Wirbelkörpers, ist eine additive ventrale endoskopische Spondylodese mit Beckenkammspan und ggf. ventraler Platte indiziert. Bei frischen stabilen osteoporotischen Wirbelfrakturen mit Kontrastmittelanreicherung in der Magnetresonanztomographie und starken Schmerzen trotz Analgetika wird eine Kyphoplastie durchgeführt.

\section{Schlüsselwörter}

Minimalinvasive Operationsmethoden . Wirbelfrakturen · Spondylodese Kyphoplastie · Fixateur interne

\section{Minimally invasive treatment of thoracolumbal spinal fractures. Options and limits}

\section{Abstract}

Minimally invasive surgery for vertebral fractures means less approach-related morbidity, decreased postoperative pain and rapid mobilization of patients. Such procedures can be performed even in elderly patients. However, along with the many advantages, minimally invasive procedures are technically demanding, require sophisticated tools and there is a learning curve for surgeons. Intraoperative visualization is often possible only radiologically and implants are generally much more expensive. Using the data from some 1,000 vertebral fracture cases treated over the past 3.5 years, we have developed a differentiated treatment concept, depending on the age of the patient and the fracture characteristics, which are presented here. Unstable fractures with involvement of the posterior edge are stabilized from a posterior approach, percutaneously with a fixator. In patients under 60 years, monoaxial screws with inserted rods (top loading) are used with which distraction and restoration of lordosis are also possible. Patients over 60 years are treated percuta- neously with a polyaxial sextant system with rods inserted to avoid avulsion of the pedicle screws from the vertebral body. To avoid cutting through the vertebra, the fenestrated screws can be augmented with cement. If a vertebral defect remains after posterior treatment, anterior fusion can also be performed endoscopically with an iliac crest bone graft and an anterior plate if necessary. In older patients, often kyphoplasty is sufficient here. For recent, stable osteoporotic fractures with enhancement of the short time inversion-recovery (STIR) T2 sequence on magnetic resonance imaging and severe pain despite analgesics kyphoplasty is performed. This is possible even in high thoracic fractures to $\mathrm{T} 3$ using thinner balloons. In $0.34 \%$ ( 2 out of 564 ) of cases post-operative neurological deficits were observed after cement extravasation.

\section{Keywords}

Surgical procedures, minimally invasive . Spinal fractures - Spondylodesis . Kyphoplasty · Fixateur interne

\section{Ventrale Spondylodese}

\section{Indikation}

Nach der dorsalen Spondylodese führen wir ein Röntgenbild und eine Compu- tertomographie (CT) durch, um die Aufrichtung des gebrochenen Wirbelkörpers zu beurteilen. In etwa 1/3 der Fälle sehen wir eine verbliebene Deckplattenimpression, bedingt durch eine darüber liegende, zerrissene Bandscheibe, die oft tief in den 


\section{Wirbelsäulenverletzungen}
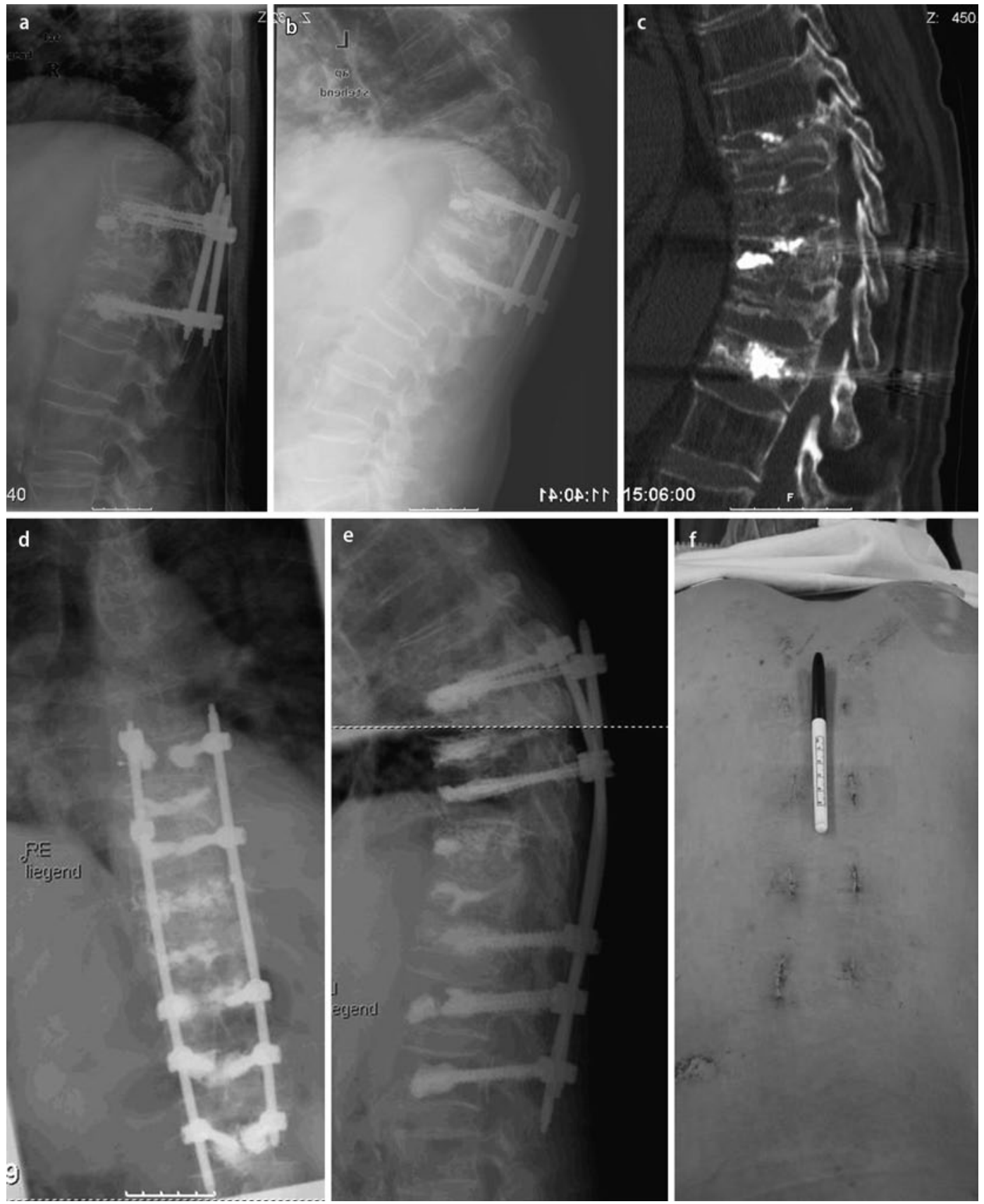

Abb. 2 \ Männlicher Patient, 81 Jahre, a postoperatives, seitliches Röntgenbild eines zementierten Fixateurs ohne Kyphoplastie, b Kontrollröntgenbild nach 6 Wochen mit $60^{\circ}$ Kyphose und Anschlussfrakturen, $\mathbf{c}$ computertomographische Kontrolle vor Revision mit Anschlussfrakturen, $\mathbf{d}$,e postoperativ nach Revision, Verlängerung des Fixateurs und Kyphoplastie, $\mathbf{f}$ Narben nach Revisionseingriff

gebrochenen Wirbel eingesprengt ist und dessen definitive Heilung verhindert. Bei einer Kneifzangenfraktur ist sogar noch die unterhalb liegende Bandscheibe mit zerstört.

Bereits 1996 konnten wir in Nachuntersuchungen von ausschließlich dorsal versorgten instabilen Frakturen aufzeigen, dass bei einem hohen Anteil die- ser derart versorgten Verletzungen 6 Monate nach Metallentfernung bzw. Abnahme des Fixateurs die kyphotische Ausgangsfehlstellung wieder auftritt. Belässt man den Fixateur, kommt es zur Lockerung, zum Bruch oder zum Ausriss des Fixateurs oder sehr selten zur spontanen Spondylodese [5]. Aus diesem Grund sehen wir eine Indikation zur ventralen Sta- bilisierung, wenn nach der dorsalen Instrumentierung ein solch tiefer Defekt der Deckplatte verbleibt.

\section{Technik}

Bis auf Höhe LWK1, z. T. auch bis LWK2, folgt in der Regel 4-6 Wochen nach der Erstversorgung die links lateral endosko- 

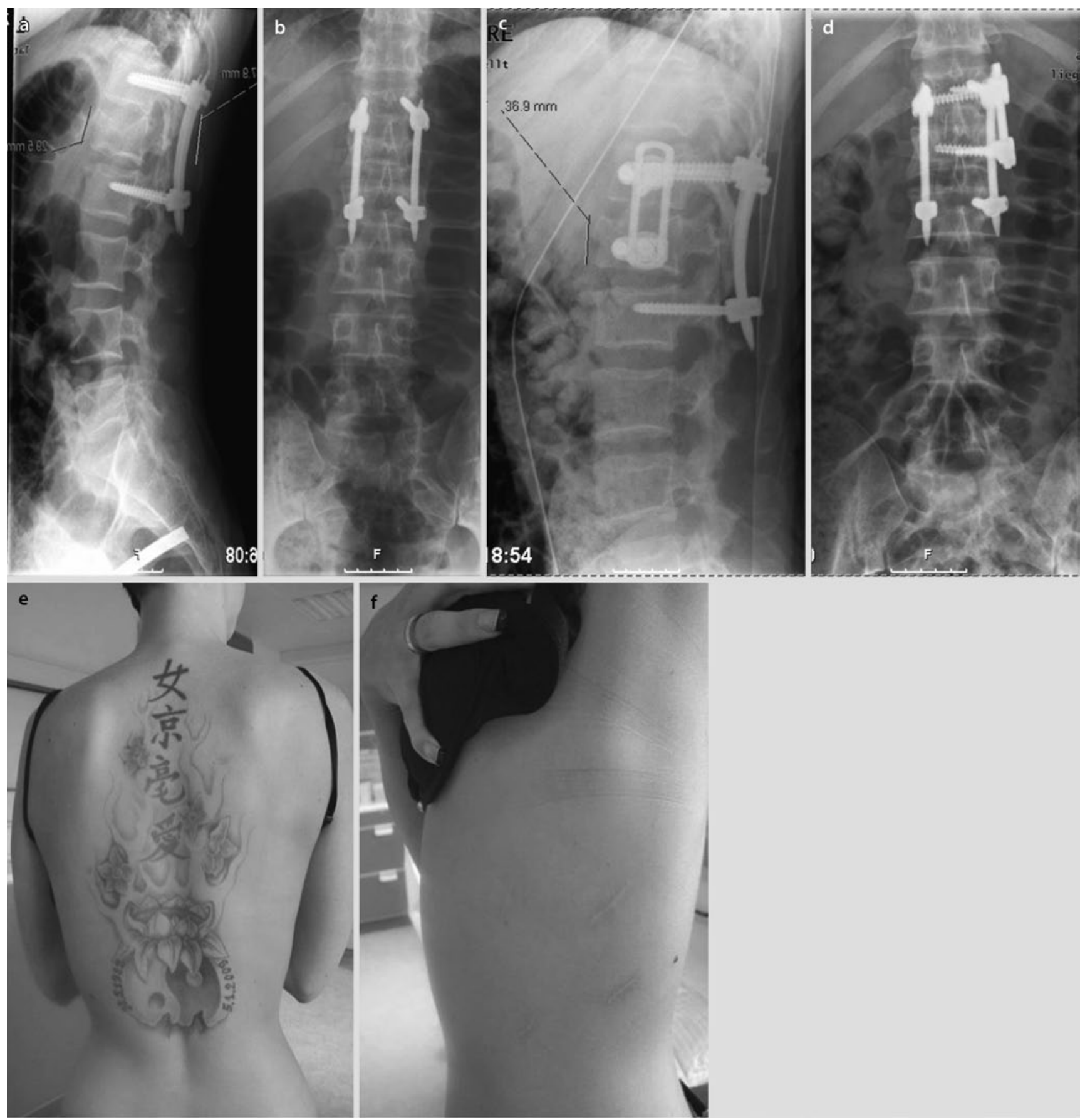

Abb. $3 \Delta$ LWK1-Fraktur Typ Wolter AB1, a,b postoperativ nach Versorgung mit Fixateur verbliebene Deckplattenimpression, c,d postoperativer Befund nach ventraler endoskopischer Spondylodese mit Span und Platte, e,f, Narben der Patientin nach dorsoventraler Versorgung

pische, minimalinvasive, transthorakale Fusion. Zwischen BWK8 und BWK3 liegt der Zugang rechts, um einen von großen Venen ungestörten Zugriff auf das laterale Bandscheibenfach zu erlangen. Zwischen LWK2 und LWK5 wird ein kleiner links retroperitonealer Zugang nach Abschiebung der Psoasmuskulatur von ventral ausgeführt. Ein transmuskulärer $\mathrm{Zu}$ - gang kann eine ungewollte Schädigung des N. femoralis bewirken. Eine Fusion LWK5/S1 (S: sakral) wird über einen pararektalen Schnitt von vorne, aber ebenfalls retroperitoneal angegangen.

Eine Aufteilung der dorsoventralen Stabilisierung auf 2 Aufenthalte hat den Vorteil, dass die Patienten besser erholt sind als beim einzeitigen Vorgehen. $\mathrm{Zu}$ - dem ist die eine einzeitige Versorgung im DRG („diagnosis related groups“) noch nicht kostendeckend abgebildet $[10,12]$.

Ist nur eine Etage betroffen, räumen wir das ganze Bandscheibenfach aus, meißeln einen Schacht im mittleren bis ventralen Drittel des betroffenen Wirbels aus und falzen unter Hyperlordosierung einen trikortikalen Beckenspan ein. 


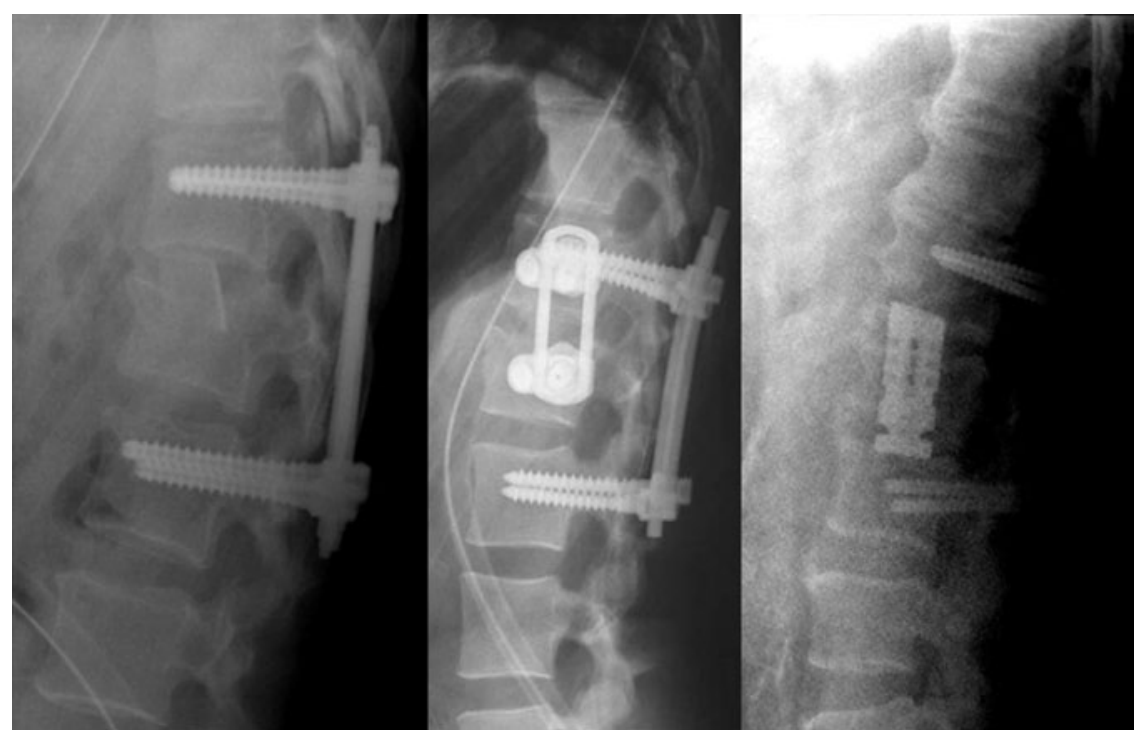

Abb. 4 \ Ventrale Spondylodese nur mit Span, ergänzender Platte und über 2 Segmente mit obelisc ${ }^{\mathrm{TM}}$

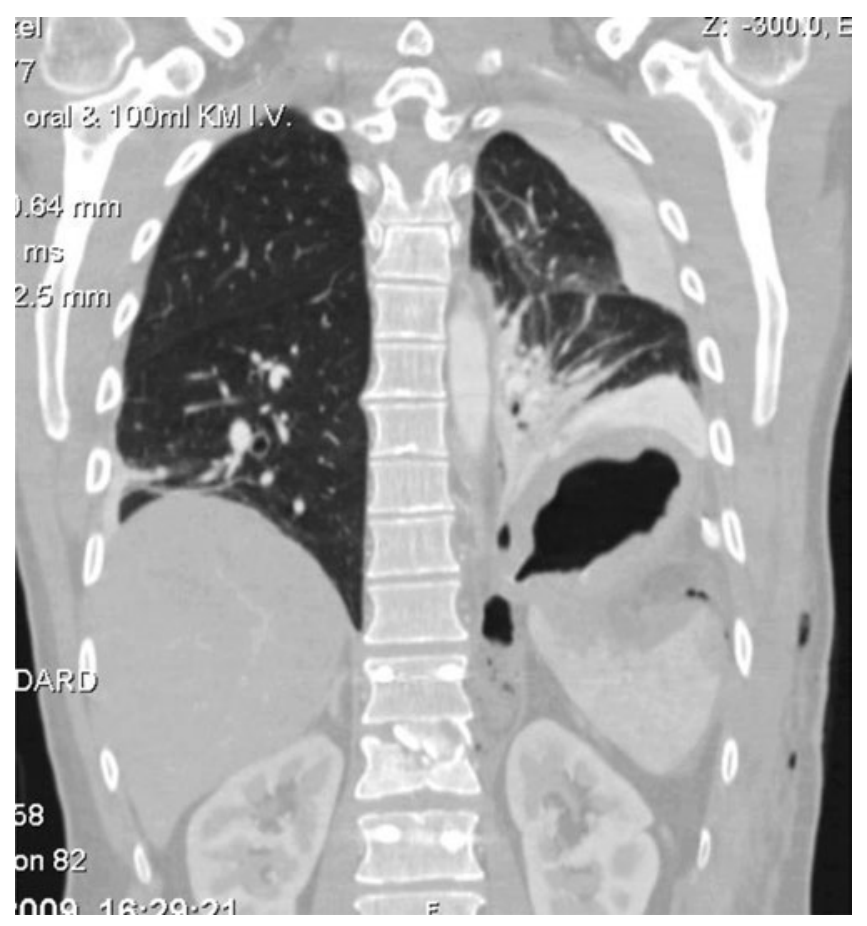

Abb. $5<$ Magenherniation transthorakal nach Zwerchfellverletzung im Rahmen der endoskopischen ventralen Operation

Kommt dieser nicht $100 \%$ pressfit zu liegen, wird darüber ein winkelstabiles Plattensystem (MACS II ${ }^{\oplus}$, Firma Aesculap) ergänzt, da es ansonsten in etwa $20 \%$ der Fälle zu einer Pseudarthrose mit unzureichender Knochenfusion kommt. Das MACS-Plattensystem ist eines der wenigen Implantate, die minimalinvasiv eingebracht werden können. Die Operationsnarben bleiben bei diesem Verfahren klein und somit kosmetisch günstig (• Abb. 3). Der Eingriff ist nicht einfach, da das gesamte Team die Instrumente und Operationsabläufe kennen muss. Ende 2011 kommt ein verbessertes System auf den Markt, welches diesbezüglich zu einer deutlichen Vereinfachung führen wird.

Bei Überbrückung von 2 Bandscheibenräumen kommt ein expandierbarer Wirbelkörperersatz (obelisc ${ }^{\mathrm{Tm}}$ der Firma Ullrich) zum Einsatz (• Abb. 4). Auch dieses Implantat kann ohne viele Schritte rein endoskopisch eingesetzt werden. Die alternative Entnahme eines Knochen- spans in dieser Größe führt bei einem hohen Prozentsatz zu Einheilungsstörungen und einer erheblichen Entnahmemorbidität. Solche Eingriffe sind nach wie vor riskant und sollten in den Händen weniger Operateure verbleiben. In unserem Haus wurden von 2008-2011 76 derartige Eingriffe von 2 verschiedenen Operateuren durchgeführt. Die Patienten, 42 Männer und 35 Frauen, wiesen ein Durchschnittsalter von 53 Jahren (21-62 Jahren) auf. Die durchschnittliche Operationszeit betrug 90 min, die mittlere Krankenhausverweildauer 13 Tage, mit fallender Tendenz.

Wichtig ist, während einer Operation auf ein eingespieltes Team von Anästhesisten, Operationsschwestern und Operateuren zurückgreifen zu können. Ein gut sitzender Doppellumentubus, der die Lunge auf der Operationsseite sicher aus der Beatmung ausschaltet, ist absolute Voraussetzung, ohne die die Operation nicht durchgeführt werden kann. Während des Eingriffs muss der Patient relaxiert sein und der Magen mittels Magensonde entlastet werden, um beim Schlitzen des Zwerchfells bei Instrumentierung von LWK1 und LWK2 eine Magenperforation zu vermeiden. Wir haben vor dieser Maßnahme in einem Fall eine Zwerchfellruptur mit Magenherniation und -perforation gesehen, der laparotomiert und versorgt werden musste (• Abb. 5). Seit dieser Zeit entlasten wir den Magen mit Sonde und verschließen Zwerchfellschlitze ab LWK1 routinemäßig endoskopisch mit einem Ankerfaden der Firma Covidien.

In einem Fall kam es zu einer neurologischen Komplikation auf Höhe Th12 durch ein spinales Hämatom, welches dorsal entlastet wurde. Regelhaft werden die Patienten noch im OP extubiert und im Aufwachraum nachkontrolliert. Ein Aufenthalt auf der Intensivstation ist nur in Ausnahmefällen erforderlich.

Bei Patienten über 65 Jahren hat die Bandscheibe einen großen Teil an Wasser verloren und ist eher als Knochen zu behandeln. Die Bandscheiben hernieren hier nicht in den gebrochenen Wirbelkörper und verhindern auch nicht, wie bei jungen Patienten, die Knochenheilung. Dies macht bei instabilen Frakturen ein anderes, weniger invasiveres Behandlungskonzept erforderlich. In diesen Fällen reicht eine Kyphoplastie nach dorsa- 

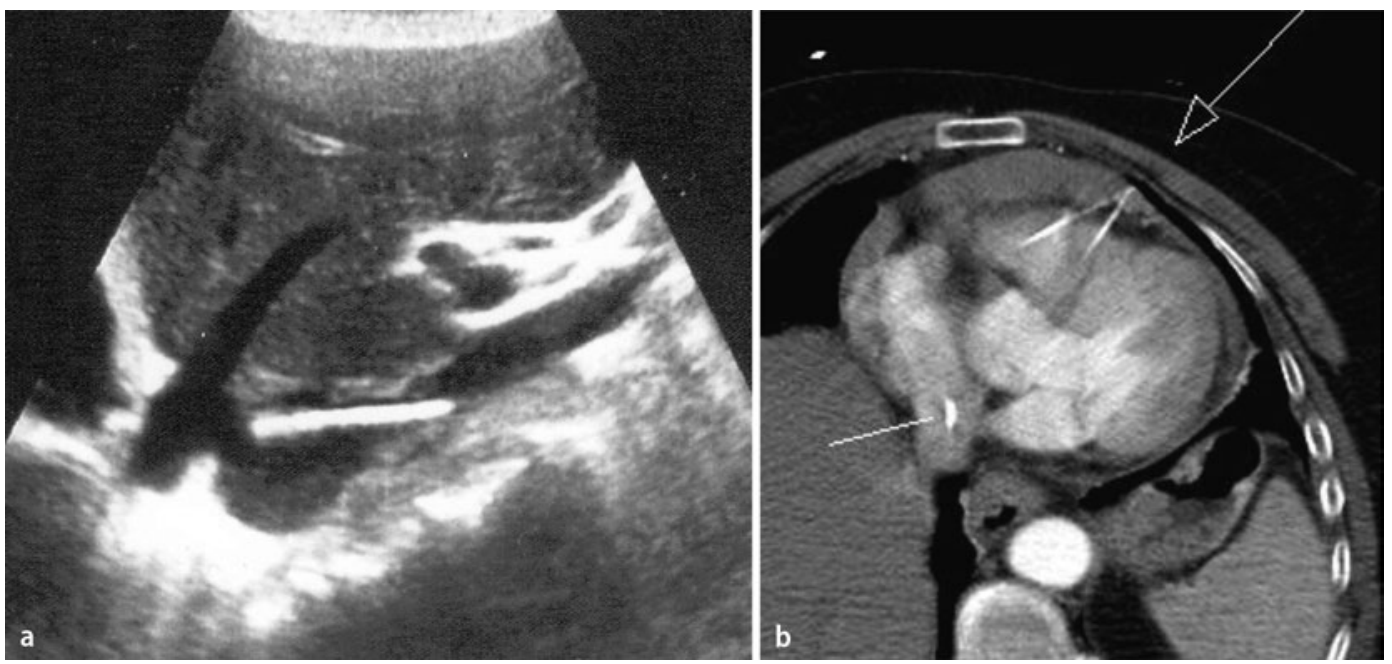

Abb. $6<$ Sonographie der oberen Hohlvene mit Pallacosembolie (a), CT mit Herzbeutelperforation und Perikarderguss durch Pallacosembolus (b)

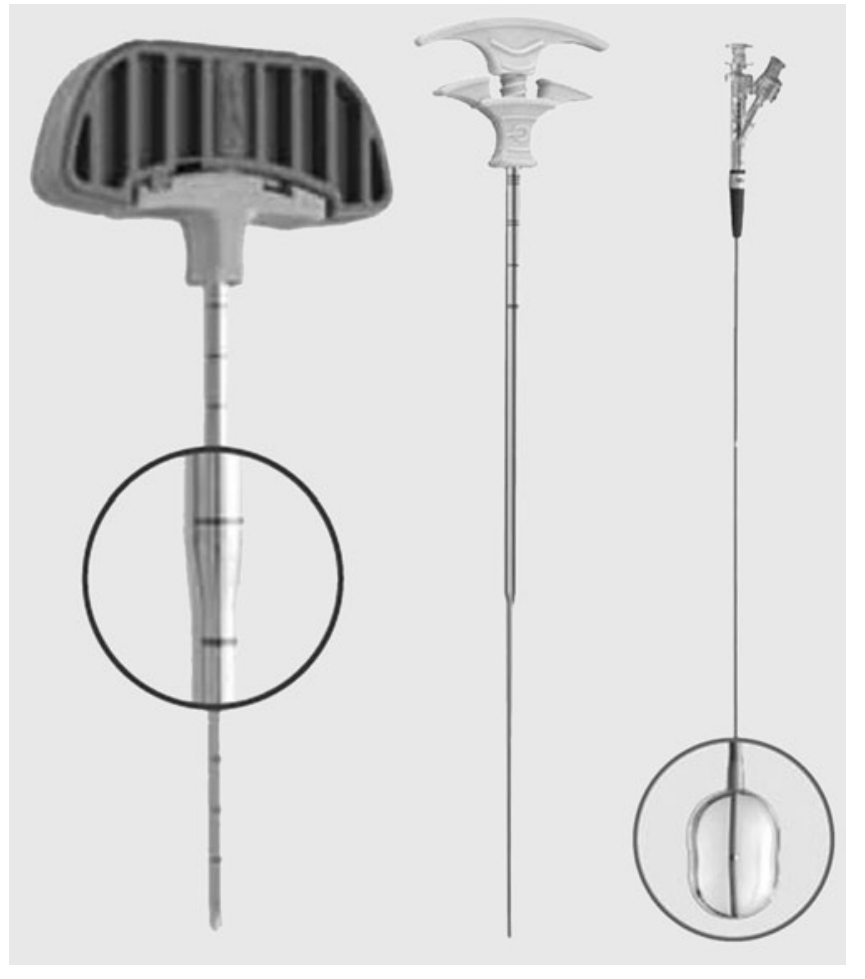

Abb. $7<$ Express-Instrumentarium für hochthorakale Versorgungen ler Instrumentierung mit zementiertem Fixateur.

Wir sind dazu übergangen, diese Kyphoplastien dann einzeitig durchzuführen, wenn eine gute Aufrichtung nach der Reposition und Instrumentierung erreicht wird. Sie werden dann im vorderen Drittel des Wirbelkörpers ausgeführt. Bei nicht guter Reposition erfolgt die Kyphoplastie zweizeitig, um einen dorsalen Austritt von Zement zu verhindern.

\section{Osteoporotische Sinterungsfraktur}

\section{Stabile schmerzhafte Frakturen}

Die hohe Prävalenz der Osteoporose führt in Deutschland zu etwa 100.000 osteoporotischen Sinterungsfrakturen der Wirbelsäule ohne relevantes Trauma pro Jahr, das entspricht etwa 5,1 Mio. Patienten bundesweit [6]. Diese Läsionen gehen mit langwierigen Schmerzen und, aufgrund der Veränderung der Statik der Wirbelsäule, einer zunehmenden Kypho- sierung einher. Dadurch steigt das Risiko weiterer Anschlussfrakturen und der Verminderung der Vitalkapazität durch Kyphosebildung.

Die Kyphoplastie ist seit über 10 Jahren eine etablierte Methode zur Behandlung von schmerzhaften osteoporotischen Sinterungsfrakturen der Wirbelsäule. Dieser Effekt wurde in mehreren klinischen Studien belegt $[1,13,18,19]$. Sie führt nicht nur zur raschen Schmerzreduktion, sondern laut einer großen Langzeitstudie auch zu einer erhöhten Überlebensrate. Edidin et al. [3] konnten über 4 Jahre 676.032 Patienten mit osteoporotischen Wirbelkörperfrakturen, die konservativ behandelt worden waren, mit 119.253 Patienten vergleichen, die kyphoplastiert, und mit 63.693, die vertebroplastiert worden waren. Die konservativ behandelten Patienten wiesen nach 4 Jahren eine 50\%ige Überlebensrate auf, während die kyphoplastierten Patienten mit 62,8\% und die vertebroplastierten mit 57,3\% signifikant länger lebten.

\section{Indikation zur Kyphoplastie}

Entsprechend der Leitlinienempfehlung ist heutzutage eine Kyphoplastie bei im MRT in der T2-STIR-Frequenz (T2: Querrelaxationszeit, STIR: „short tau inversion recovery“) nachgewiesener frischer Fraktur mit starken, über 6 Wochen vergeblich konservativ behandelten Schmerzen indiziert. Die guten klinischen Ergebnisse mit sofortiger Schmerzreduktion, schnellerer Mobilität und inzwischen nachgewiesener besserer Lang- 

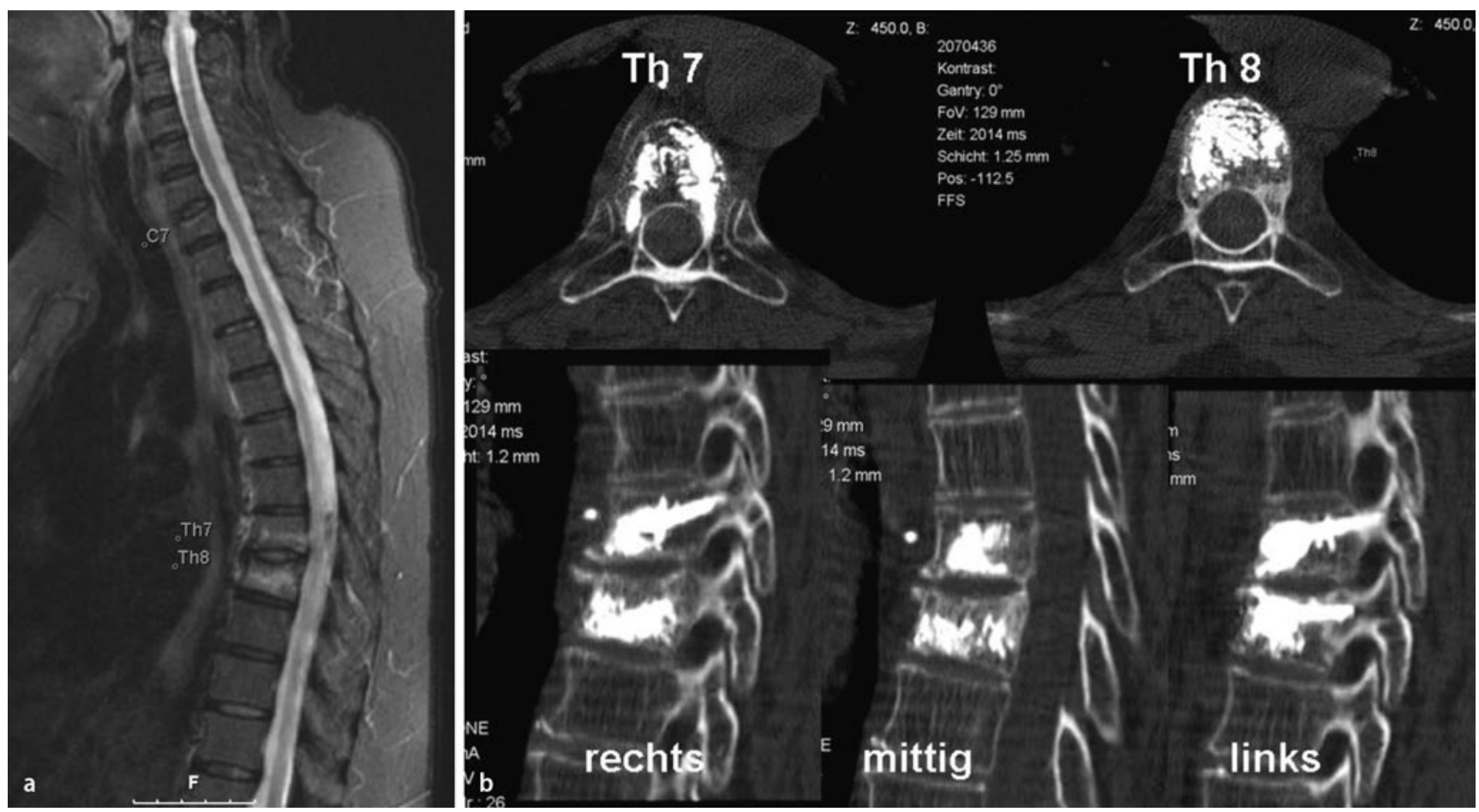

Abb. $8 \Delta$ Osteoporotische schmerzhafte Sinterungsfraktur in Höhe BWK7 und BWK8, a präoperatives MRT, T2 STIR, b postoperatives CT nach Kyphoplastie

zeitprognose werden diese Empfehlung in Zukunft relativieren.

Wir klären die Patienten ausführlich über operative und konservative Behandlungen auf und kyphoplastieren bei im MRT nachgewiesener frischer Fraktur bei einer Schmerzangabe von über 6 Schmerzpunkten in der visuellen Analogskala.

\section{Ergebnisse nach Kyphoplastie}

Vom 01.01.2008 bis zum 01.08.2011 wurden bei 564 Patienten ballonassistierte Kyphoplastien (Kyphoplastie, Express, Vertebro Body Stent, Vesseloplastie) durchgeführt. In allen Fällen bestanden Schmerzen von 6 und mehr Schmerzpunkten und eine nachgewiesene frische Fraktur sowohl in einer Röntgenaufnahme der Wirbelsäule als auch einem ergänzenden Schnittbildverfahren (CT oder MRT STIR T2; $[9,13])$.

Das Durchschnittsalter betrug 75,3 Jahre. In 71,3\% der Fälle waren Frauen betroffen. Es wurden Frakturen von BWK3 bis LWK5 behandelt. In 372 Fällen wurde 1 Etage, in 128 Fällen 2 Etagen, in 48 Fällen 3 Etagen und in 22 Fällen 4 Etagen simultan versorgt. Die mittlere Operationszeit bei allen Patienten betrug
36 min. Die Eingriffe wurden von 8 verschiedenen Operateuren ausgeführt. Die mittlere Liegezeit betrug 8 Tage und war über die Jahre stetig fallend.

Die Operationen wurden nach $\mathrm{Ab}$ laufoptimierung kostendeckend durchgeführt $[11,15]$. Es traten 6 Hauptkomplikationen $(1,06 \%)$ auf:

- In 2 Fällen kam es zu neurologischen Ausfällen durch dorsale Zementparavasate $(0,34 \%)$, in einem Fall auf Höhe BWK8 mit inkompletter Halbseitenparese und spontaner, vollständiger Remission nach 3 Monaten und in einem Fall auf Höhe $\mathrm{LWK}_{3}$ nach Fehlpunktion mit einer persistierenden Hüftbeugerparese Kraftgrad 4.

- 2 Patienten verstarben während des stationären Aufenthalts (weiblich, 93 Jahre, Herzversagen 4 Tage postoperativ nach beschwerdefreier Mobilisation; weiblich, 78 Jahre, kardiopulmonales Versagen 26 Tage postoperativ).

- In einem Fall (weiblich, 61 Jahre, LWK1) kam es zum schmerzhaften Ausbruch eines Stents nach lateral. Er wurde endoskopisch transthorakal geborgen. Bei der Thorakoskopie kam es zur Zwerchfellruptur und
Magenherniation, sodass notfallmäßig eine Laparotomie und Zwerchfellnaht erforderlich waren. Die Patientin ist heute beschwerdefrei.

- Bei einer Patientin kam es zu einem ausgedehnten, anfänglich asymptomatischen Zementaustritt in Lungen und Hohlvenen. 2,5 Jahre später löste sich ein Zementembolus, der zu einer akuten Herzperforation mit notfallmäßiger Thorakotomie führte (• Abb. 6). Der Herzbeutel wurde genäht, die Patientin überlebte und ist heute beschwerdefrei.

\section{Sonderfälle hochthorakaler osteoporotischer Sinterungs- frakturen oberhalb BWK8}

\section{Problematik}

Bei Frakturen oberhalb von BWK8 wird die Kyphoplastie technisch anspruchsvoller. Kleinere Pedikel und Wirbelkörper sowie die Überlagerung von Lunge und Armen im intraoperativen Röntgenbild erschweren die Operation. Allerdings liegt der Anteil hochthorakaler osteoporotischer Frakturen an allen osteoporotischen Wirbelbrüchen zwischen 20 und 30\%. Das Risiko einer Fehlpunktion 
oder eines dorsalen Zementaustritts mit neurologischen Ausfällen nimmt in der oberen Brustwirbelsäule deutlich zu.

Inzwischen wurden neue Instrumentarien mit einer 30\% kleineren Punktionsnadel und angepassten kleineren Ballons entwickelt, die auch in der oberen Brustwirbelsäule einen sicheren transpedikulären Zugang ermöglichen (Express, Medtronic, 0 Abb. 7). Sie stehen in Europa seit dem 01.09.2009 zur Verfügung.

\section{Ergebnisse}

Bis zum 01.06.2011 konnten wir $66 \mathrm{~Pa}$ tienten mit Verletzungen im Bereich von BWK3 bis BWK8 mit dem ExpressSystem versorgen. Es handelte sich um 43 Frauen und 23 Männer, sie wiesen ein Durchschnittsalter von 75 Jahren und eine ASA-Klassifikation Stadium II-III auf.

Die Operationszeiten betrugen im Mittel 26 min bei einer Durchleuchtungszeit von 2,7 min pro Patient. Es kam zu 12 lateralen und ventralen und $\mathrm{zu} 4$ dorsalen asymptomatischen Paravasaten. Die Schmerzen, bemessen nach der visuellen Analogskala, verbesserten sich von 8,1 Punkten präoperativ auf 2,3 Punkte postoperativ. Die durchschnittliche Krankenhausliegezeit betrug 5 Tage. Nach 6 Monaten wurden 40 Patienten nachuntersucht. Trotz medikamentöser Prophylaxe kam es 6-mal zu Anschlussfrakturen. Die Schmerzen betrugen im Mittel 2,2 Punkte.

Eine Kyphoplastie war mit dem Express-Instrumentarium bis BWK3 gut möglich, allerdings sollten hier standardmäßig ein Karbontisch und ein sehr gutes Röntgengerät mit der Möglichkeit einer intraoperativen 3D-Rekonstruktion angewendet werden. Aufgrund der kleineren Wirbelkörper reichen kleine Zementmengen von 1-2 $\mathrm{ml}$ pro Seite aus ([16], - Abb. 8).

\section{Fazit für die Praxis}

Eine Stabilisierung instabiler Frakturen ist minimalinvasiv über perkutane dorsale Fixateure möglich. Sie ist schnell, sicher, ohne großen Blutverlust und ohne Störung der Muskulatur durchführbar. Bei fortbestehender Impression der
Deckplatte ist bei jungen Patienten eine minimalinvasive ventrale Spondylodese zu ergänzen. Bei Patienten über 60 Jahre mit instabilen Frakturen sollten zementaugmentierte Fixateure eingesetzt werden, um den Halt im Knochen zu verbessern. Die gebrochenen Wirbel können in diesen Fällen ein- oder zweizeitig mit Kyphoplastien sicher stabilisiert werden. Stabile schmerzhafte osteoporotische Sinterungsfrakturen lassen sich mit sehr gutem Erfolg kyphoplastieren und ab BWK8 aufwärts mit kleineren Ballonsystemen bis auf Höhe BWK3 durch erfahrene Operateure stabilisieren.

\section{Korrespondenzadresse}

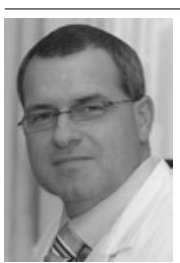

Prof. Dr. A. Prokop

Unfallchirurgie,

Kliniken Sindelfingen,

Klinikverbund Südwest,

Arthur-Gruber-Straße 70,

71065 Sindelfingen

verletzt@klinikverbundsuedwest.de

Interessenkonflikt. Der korrespondierende Autor gibt an, dass kein Interessenkonflikt besteht.

The supplement containing this article is not sponsored by industry.

\section{Literatur}

1. Bouza C, Lopez T, Magro A et al (2006) Efficacy and saftey of balloon kyphoplasty in the treatment of vertebral compression fractures: a systemativ review. Eur Spine J 15:1050-1067

2. Chmielnicki M, Stenz R, Prokop A (2010) Unstable vertebral body fractures in elderly patients. How stable is percutaneous internal fixation? J Orthop 7:1-10

3. Edidin AA, Ong KL, Lau E, Kurtz SM (2011) Mortality risk for operated and non-operated vertebral fracture patients in the medicare population. J Bone Miner Res 26(7):1617-1626. DOI 10.1002/ jbmr.353

4. Gejo R, Matsui $H$, Kawaguchi Y et al (1999) Serial changes in trunc muscle performance after posterior lumbar surgery. Spine 24:1023-1028

5. Helling HJ, Prokop A, Ullrich G et al (1996) CT-gestützte Morphometrie nach dorsaler Instrumentation bei instabilen Wirbelkörperbrüchen. Hefte Unfallchir 262:196

6. Kasperk C, Nöldgen G, Meeder P et al (2008) Kyphoplastie. Minimal-invasives Verfahren zur Versorgung schmerzhafter Wirbelkörperfrakturen. Chirurg 79:944-955

7. Prokop A, Löhlein F, Chmielnicki M, Volbracht J (2008) Minimally invasive dorsale instrumentation with spine fractures. More expensive or even better? Eur Spine J 17:1612-1613
8. Prokop A, Löhlein F, Chmielnicki M, Volbracht J (2009) Minimal invasive perkutane Instrumentation bei Wirbelsäulenfrakturen. Unfallchirurg 112:621-628

9. Prokop A, Stenz R, Löhlein F et al (2009) Vesselplasty - new method in treatment of osteoporotic spine fractures. Langenbecks Arch Surg 394:965

10. Prokop A, Wollny M, Futterer N et al (2009) Operative Versorgung von instabilen osteoporotischen Wirbelsäulenfrakturen. Eine wichtige Kodierhilfe. Unfallchirurg 112:815-819

11. Prokop A, Chmielnicki M, Greiling M (2010) Analysis of costs in kyphoplasty. Eur Spine J 19:2060

12. Prokop A, Chmielnicki M, Volbracht J (2010) DRG 2010 bei Wirbelfrakturen. Orthop Nachricht 10:1718

13. Prokop A, Löhlein F, Chmielnicki M et al (2010) Kyphoplastie bei osteoporotischen Wirbelsäulenfrakturen. Unfallchirurg 113:127-132

14. Prokop A, Reinauer KM, Futterer N et al (2010) Lohnt sich eine Zertifizierung in der Unfallchirurgie? Erfahrungen aus einem zertifizierten Zentrum für Alterstraumatologie. DGU Mitteil Nachricht 62:18-19

15. Prokop A, Berner U, Muszynski T et al (2011) Prozess-Kostenanalyse von Kyphoplastien bei osteoporotischen Sinterungsfrakturen. Unfallchirurg 114:248-250

16. Prokop A, Chmilnicki M, Stenz R, Berner U (2011) Kyphoplastie bei hoch thorakalen osteoporotischen Wirbelfrakturen. Chir Prax 73:37-46

17. Sihvonen T, Herno A, Paljarvi L (1993) Local denervation atrophy of paraspinal muscles in postoperative failed back syndrome. Spine 18:575-581

18. Taylor RS, Fritzell P, Taylor RJ (2011) Balloon kyphoplasty in the management of vertebral compression fractures: an updated systematic review and meta-analysis. Eur Spine J 16(8):1085-1100. DOI 10.1007/s00586-007-0308-z

19. Wardlow D, Cummings SR, Meirhaeghe JV et al (2009) Efficacy and safety of balloon kyphoplasty compared with non-surgical for vertebral compression fracture (FREE): a randomised controlled trial. Lancet 373:1016-1024 